\title{
MULTIPLE GIANT ANEURYSMS IN KAWASAKI DISEASE
}

Beatriz Toledo Mendes ${ }^{1}$, Bruna de Paula Gonçalves Sousa Lyraํㅜ, Clara Demeneck Pereira ${ }^{1}$, Luciana Oliveira Castro e Silva Sobral ${ }^{2}$, Maria Clara Potiguara Azevedo Teixeira ${ }^{1, *}$, Mariana Oliveira Santana ${ }^{1}$, Mirella Bastos Sales ${ }^{1}$

1. Centro Universitário de Brasília, Brasília (DF), Brazil. 2.Hospital da Criança de Brasília, Brasília (DF), Brazil.

*Corresponding author: mclaraatx@sempreceub.com

\section{BACKGROUND}

The Kawasaki disease is a systemic vasculitis in medium caliber vessels with self-limited course and coronary arteries predilection, with aneurysms' formation. The physiopathology involves inflammatory changes in the medial layer with edema and cells necrosis, compromising the structural integrity with the formation of aneurysmatic dilations. In the pediatric population, it is a concern because they are associated with morbidity and mortality with the associated risk of thrombosis, infarction and sudden death. The therapeutics preconizes a reduction in the tissue inflammatory process, based on the use of immunoglobulin and acetylsalicylic acid (ASA) as an antiplatelet agent, with the option of combining corticosteroid therapy or an alternative treatment such as cyclosporine, cyclophosphamide and plasma exchange.

\section{CASE REPORT}

B.B.A., 1 year and 3 months of age, previously healthy, hospitalized with suspicion of incomplete Kawasaki disease, had high fever and urticarial rash on the trunk and limbs. On the 3rd day of fever, he developed edema and hyperemia of hands and feet, and slight periungual desquamation. A pediatric Doppler echocardiogram (ECO) was performed, which showed cardiac involvement with the presence of coronary aneurysms: left coronary (LC): $z+6.5$, anterior descending (AD): $z+3$ and right coronary $(R C): z+1,5$. Immunoglobulin, cycles of methylprednisolone, infliximab and AAS were prescribed in association with cyclosporine. Upon examination, cardiovascular apparatus without audible murmurs, normophonetic sounds. Respiratory system without dizziness. Abdomen without visceromegalies. Therefore, no signs of congestive heart failure. He presented with urinary tract infection and ceftriaxone and cephalexin were added to the therapeutic regimen. In the control ECO, coronary dilation was noted (LC: $z+8.89$, $A D: z+6.5$ and $R C:+19.1$ ) and the presence of mild pericardial effusion. The use of beta-blockers and enoxaparin was started. On outpatient return, it was observed that, over the months, the infant had a good evolution with the prescribed medications, remaining hemodynamically stable. In a new ECO, a gradual reduction in aneurysms was noticeable: LC: $z+3.4, A D: z+1.8$ and RC: $z+14$.

\section{CONCLUSION}

Cardiovascular complications represent the main contributors to Kawasaki disease-related morbidity and mortality, both in acute and long-term illness in the chronic phase. Early diagnosis and appropriate treatment can directly interfere with the outcome of the disease. 